Published in final edited form as:

Science. 2013 August 9; 341(6146): 673-677. doi:10.1126/science.1241680.

\title{
T Follicular Helper Cell Dynamics in Germinal Centers
}

\author{
Ziv Shulman ${ }^{1}$, Alexander D. Gitlin ${ }^{1}$, Sasha Targ $^{3}$, Mila Jankovic ${ }^{1}$, Giulia Pasqual ${ }^{3}$, Michel C. \\ Nussenzweig ${ }^{1,2, *}$, and Gabriel D. Victora ${ }^{3,{ }^{*}}$ \\ ${ }^{1}$ Laboratory of Molecular Immunology, The Rockefeller University, New York, New York 10021, \\ USA \\ ${ }^{2}$ Howard Hughes Medical Institute, The Rockefeller University, New York, New York 10021, USA \\ ${ }^{3}$ Whitehead Institute for Biomedical Research, Cambridge, MA, 02138, USA
}

\begin{abstract}
$\mathrm{T}$ follicular helper cells $\left(\mathrm{T}_{\mathrm{FH}}\right)$ are a specialized subset of effector $\mathrm{T}$ cells that provide help to and thereby select high-affinity B cells in germinal centers (GCs). To examine the dynamic behavior of $\mathrm{T}_{\mathrm{FH}}$ cells in GCs in mice we combined two-photon microscopy and optical highlighting using a photoactivatable fluorescent reporter. Unlike GC B cells, which are clonally restricted, $\mathrm{T}_{\mathrm{FH}}$ distributed among all GCs in lymph nodes and continually emigrated into the follicle and neighboring GCs. Moreover, newly activated $\mathrm{T}_{\mathrm{FH}}$ cells invaded pre-existing GCs, where they contributed to $\mathrm{B}$ cell selection and plasmablast differentiation. Our data suggest that dynamic exchange of $\mathrm{T}_{\mathrm{FH}}$ between GCs ensures maximal diversification of $\mathrm{T}$ cell help and their ability to enter ongoing GCs accommodates antigenic variation during the immune response.
\end{abstract}

T cells play a pivotal role in affinity maturation by selecting B cells to enter the GC, regulating GC positive selection, and directing $\mathrm{B}$ cell differentiation to plasma cells and memory B cells (1-6). These events are orchestrated by a specialized population of GCresident $\mathrm{T}$ follicular helper $\left(\mathrm{GC}-\mathrm{T}_{\mathrm{FH}}\right)$ cells that develop in concert with $\mathrm{GC}$ B cells (7-13). $\mathrm{T}_{\mathrm{FH}}$ cells express chemokine receptor CXCR5, CD28 family members ICOS and PD-1, transcription factor Bcl-6, and cytokines interleukin (IL)-4 and IL-21, many of which are required for GC-B cell survival and differentiation (7). Less is known about the dynamic properties of $\mathrm{T}_{\mathrm{FH}}$ cells and how these might affect GC B cell selection.

To document the kinetics of $\mathrm{T}$ cell expansion and localization during the $\mathrm{GC}$ reaction, we examined whole-mounted lymph nodes using two-photon laser scanning microscopy (TPSLM) $(14,15)$. In agreement with previous reports $(16)$, large numbers of antigenspecific $\mathrm{T}$ cells were found in the $\mathrm{T}$ cell zone 3 days after immunization, which then spread throughout the lymph node, including B cell follicles and nascent GCs by day 5 (Fig. 1A). T cells only began to concentrate in GCs after 8-11 days (Fig. 1A) coinciding with the peak of T cell help to GC B cells (5). Thus, unlike B cell clones, which are thought to expand in a

Address correspondence to: Michel C Nussenzweig, Laboratory of Molecular Immunology, Rockefeller University, 1230 York Avenue, New York, NY, 10065, nussen@ rockefeller.edu, and, Gabriel D Victora, Whitehead Institute for Biomedical Research, Nine Cambridge Center, Cambridge, MA, 02142, victora@wi.mit.edu.

these authors contributed equally

Supplementary Materials

Materials and Methods

References (25-29)

Supplementary Figures S1-S8

Supplementary Table S1

Movie Legends

Movies S1-S7 
confined micro-anatomical region to produce pauciclonal GCs $(17,18)$, responding T cells are initially evenly distributed throughout the entire lymph node, and accumulate in GCs only after these have coalesced.

$\mathrm{T}_{\mathrm{FH}}$ and GC- $\mathrm{T}_{\mathrm{FH}}$ are commonly defined based on functional properties and the expression of cell surface markers $(8,19)$, rather than on anatomical localization. To verify the correspondence between surface phenotype and microanatomical location, we labeled cells within spatially restricted areas using photoactivatable GFP (PAGFP) (3). Flow-cytometric analysis of photoactivated OT-II T cells (Fig. S1A-C) showed that CXCR5 and PD-1 expression were highest among cells physically inside the GC and lowest among T cells in the paracortex, whereas T cells in the follicle outside the GC showed intermediate levels of expression of these molecules (Fig. 1B-C and fig. S1D). In contrast, ICOS expression was comparable in all three locations (Fig. 1B-C and fig. S1D). Similar results were obtained by photoactivation of endogenous polyclonal T cells (Fig. S2). Thus, although CXCR5 and PD-1 expression distinguish between GC- $\mathrm{T}_{\mathrm{FH}}$ and paracortical $\mathrm{T}$ cells, they cannot be used to definitively distinguish follicular $\mathrm{T}_{\mathrm{FH}}$ from either of these populations.

To determine whether GC- $\mathrm{T}_{\mathrm{FH}}$ cells are clonally restricted within individual GCs like their B cell counterparts $(17,18)$, we immunized mice that had received a mixture of OT-II T cells expressing one of three fluorescent proteins and visualized GCs 10 days after immunization (Fig. 2A). As suggested by our initial observations, the proportion of $\mathrm{T}$ cells of each color within individual GCs was constant across all GCs in the same lymph node (LN), indicating that $\mathrm{T}$ cells are not clonally restricted within GCs (Fig. 2A-B and fig. S3A).

This pattern of $\mathrm{T}$ cell distribution might result from initial colonization by multiple clones, and/or from T cell exchange between GCs. To examine the first of these possibilities, we analyzed early GCs. GC- $\mathrm{T}_{\mathrm{FH}}$ distribution was homogeneous across $\mathrm{GCs}$ even at the earliest observable time point, suggesting that individual GCs are indeed colonized by several distinct $\mathrm{T}$ cell clones (Fig. $2 \mathrm{C}$ and Fig. S3B-C). We confirmed this by transferring a much smaller number of T cells ( $3 \times 10^{4}$ total T cells per mouse) at a 19:1 ratio of GFP:DsRed cells (Fig. S4), as well as by reconstituting TCR $\beta$-deficient mice with mixtures of geneticallylabeled polyclonal $\mathrm{CD}^{+} \mathrm{T}$ cells (Fig. S5). In both cases, $\mathrm{T}$ cells were evenly distributed across GCs after immunization (Fig. S4 and S5). Thus, we find no evidence that GC- $\mathrm{T}_{\mathrm{FH}}$ are clonally restricted to individual GCs; instead, GCs appear to be colonized by a heterogeneous population of $\mathrm{T}$ cells representing all responding $\mathrm{T}$ cell clones.

To determine whether GC- $\mathrm{T}_{\mathrm{FH}}$ cells can also exchange between GCs by migration, we imaged these cells by TPLSM. Short-term intravital movies showed that GC- $\mathrm{T}_{\mathrm{FH}}$ occasionally migrated out of GCs (Movie S1). Whether these emigrants are in fact leaving the GC rather than making a transient foray into the follicle could not be determined due to constraints in the duration of conventional intravital imaging movies (20). To observe GC$\mathrm{T}_{\mathrm{FH}}$ migration for longer periods, we therefore photoactivated PAGFP-OT-II $\mathrm{T}_{\mathrm{FH}}$ within single GCs (Fig. S6A). Photoactivation does not alter the motility of PAGFP ${ }^{+}$OT-II cells compared to controls (Movie S2), and, because $\mathrm{GC}_{-} \mathrm{T}_{\mathrm{FH}}$ are in $\mathrm{G} 1$ phase (Fig. S6B), activated PAGFP is not diluted by cell division, allowing for long-term cell tracking. Immediately after photoactivation, $\mathrm{PAGFP}^{+} \mathrm{OT}-\mathrm{II}$ cells were restricted to the targeted region (Fig. S6C). In contrast, $\sim 20$ hours after activation, 32\% of photoactivated GC-T $\mathrm{FH}_{\mathrm{FH}}$ cells were found outside the original GC (Fig. 3A-B). Emigrating cells were found almost entirely within neighboring follicles and other GCs, and only very rarely ( 1 cell out of 487 analyzed) in the T cell zone (Fig. 3B and S6D, Movie S3). Consistent with their near absence from the $\mathrm{T}$ cell zone, we could detect only a very small number of photoactivated cells in the blood or pooled distal lymphoid organs of mice examined 36 hours after photoactivation (1-4 cells per mouse, 7 mice in three independent experiments, Fig. S6E). 
Transfer of $\mathrm{PAGFP}^{+} \mathrm{OT}$-II T cells before OVA priming did not substantially alter GC size or the ratio of T to B cells in the GC (Fig. S6F-G); thus, GC- $\mathrm{T}_{\mathrm{FH}}$ cell emigration to the follicle and neighboring GCs cannot be attributed to a non-physiological density of $\mathrm{T}_{\mathrm{FH}}$ cells.

To determine the phenotype of emigrant $\mathrm{GC}-\mathrm{T}_{\mathrm{FH}}$ cells, we microdissected lymph nodes into two fragments, one of which contained the original photoactivated GC (Fig. 3C and S7AB). Immediately after activation (0 hours), the lymph node fragment contralateral to the activated GC contain no photoactivated cells (Fig. S7C). In contrast, after 24 hours, photoactivated cells corresponding to $\mathrm{GC}-\mathrm{T}_{\mathrm{FH}}$ that had emigrated from the original $\mathrm{GC}$ could also be found in the non-photoactivated half of the lymph node (Fig. 3C). In agreement with the lymph node scans (Fig. 3A), emigrant $\mathrm{GC}_{\mathrm{T}} \mathrm{T}_{\mathrm{FH}}$ cells remained phenotypically similar to authentic GC- $\mathrm{T}_{\mathrm{FH}}$, although expression of PD-1 and CXCR5 was slightly decreased (Fig. 3C and fig. S7D-E). We conclude that GC-T $\mathrm{THH}_{\mathrm{FH}}$ emigrate and redistribute throughout the follicles and to neighboring GCs, but only rarely leave the follicle to enter circulation in the timeframe analyzed.

The exchange of $\mathrm{T}$ cells between GCs suggested a hitherto unappreciated dynamic equilibrium between $\mathrm{T}$ cells in the follicles and in different GCs. We reasoned that, if GCs are in fact open to $\mathrm{T}_{\mathrm{FH}}$ exchange, then newly-activated $\mathrm{T}$ cells may also be able to join an ongoing GC reaction. To examine this possibility, we transferred GFP-expressing OT-II cells into WT recipients that were then primed with OVA to induce OT-II expansion. These mice then received CFP-expressing B1-8hi cells, and were immunized with NP-CGG in alum to produce GCs in which endogenous CGG specific $\mathrm{T}_{\mathrm{FH}}$ provide help for B1-8 ${ }^{\text {hi }}$-CFP. The immunized mice were then boosted subcutaneously with soluble NP-OVA to trigger invasion of these GCs by OT-II cells (Fig. S8A). Before the boost, small numbers of GFPOT-II cells were found scattered throughout the $\mathrm{T}$ cell zone, occasionally entering existing GCs for brief periods (Fig. 4A and fig. S8B; Movie S4). One day after immunization, GFP ${ }^{+}$ cells could be seen proliferating in foci of T and B cells at the follicle/T zone border (Fig. 4A, Movie S5). By day 3 after boost, GFP-OT-II cells were found throughout the lymph node follicles and within pre-existing GCs (Fig. 4A, Movie S6). All GCs evaluated contained invading GFP-OT-II cells, indicating that these cells were indeed entering existing GCs rather than producing GCs de novo. Accordingly, control mice boosted with NP-OVA without pre-immunization with NP-CGG had no GCs at this time point (Fig. S8C). Over time, GFP-OT-II cells began to accumulate progressively within GCs, so that by day 11 after boost, the newly activated $\mathrm{T}$ cells were mostly concentrated inside these structures (Fig. 4A and S8B; Movie S6), interacting actively with GC B cells (Fig. 4B; Movie S7). Therefore, ongoing GCs are open to invasion by newly-activated $\mathrm{T}_{\mathrm{FH}}$.

To determine whether invading $\mathrm{T}_{\mathrm{FH}}$ contribute to the $\mathrm{GC}$ reaction, we measured their ability to provide help to $\mathrm{B}$ cells selectively expressing high levels of cognate peptide-MHC by targeting OVA to GC B cells using antibodies to DEC-205 (encoded by Ly75) (3, 4). OVAspecific GC- $\mathrm{T}_{\mathrm{FH}}$ cells were induced to invade a CGG-specific GC containing a mixture of 85\% Ly $75^{-/-}$CD $45.1 / 2$ and $15 \%$ Ly $75^{+/+}$CD $45.1 / 1$ B1- ${ }^{\text {hi }}$ B cells, and cognate MHCpeptide expression was artificially increased on the $L y 75^{+/+} \mathrm{B}$ cells by injection of antiDEC205-OVA antibodies (Fig. S8D). Whereas PBS or control antibody had no effect, antiDEC205-OVA injection resulted in selective and dramatic expansion of the $L y 75^{+/+}$but not $L y 75^{-/-}$GC B cells, and a nearly 10 -fold increase in plasmablast frequency (Fig. 4C-D). We conclude that $\mathrm{T}$ cells that invade ongoing GCs actively participate as helper $\mathrm{T}$ cells and positively select B cells expressing high levels of MHC-peptide.

Boosting ongoing NP-CGG-specific GCs with NP-OVA (as detailed in Fig. 4A) resulted in a five-fold increase in the proportion of GC B cells five days after boost (from $0.9 \%$ to 
$5.1 \%$ ), while the proportion of transferred to endogenous B cells remained constant (Fig. $4 \mathrm{E})$. Thus, $\mathrm{GC}$ invasion by newly-activated $\mathrm{T}_{\mathrm{FH}}$ cells can augment ongoing $\mathrm{GC}$ reactions.

Physical restriction of responding B cells to a single GC minimizes competition between B cells in different GCs, thereby preventing the immune response from converging on a single dominant clone (21). In contrast, free movement of $\mathrm{T}_{\mathrm{FH}}$ cells between GCs may be advantageous, in that it ensures diversified and robust support for B cell clonal expansion and affinity maturation (22-24). Thus, diversity in the antibody response appears to be favored by restricting B cell clones to single GCs while exposing them to a multiplicity of different $\mathrm{T}$ cell clones that transit between different GCs.

In addition to polyclonal colonization and dynamic exchange between GCs, newly-activated $\mathrm{T}$ cells can also enter ongoing GCs. This raises the possibility that $\mathrm{T}$ cells that are activated late in the immune response can join and potentially prolong the GC reaction, a feature that may be a significant advantage in responding to chronic infections and/or pathogens that diversify their antigenic epitopes during infection, such as HIV.

\section{Materials and Methods}

\section{Mice}

Wild-type C57BL6, B6.SJL (CD45.1 $\left.1^{+}\right), T c r b^{-/-}$, and transgenic mice ubiquitously expressing CFP, DsRed, or GFP were purchased from Jackson Laboratories. Fluorescent strains were bred to OT-II TCR-transgenic (Y chromosome) mice in our laboratories. PAGFP-transgenic (3), B1-8 ${ }^{\text {hi }}$ knock-in (25) and $L y 75^{-/-}$(DEC205-deficient) mice (26) were generated and maintained in our laboratories. MHC-II-GFP mice (27) were generated and maintained at the Whitehead Institute animal facility. Mice ubiquitously expressing tdTomato were generated by crossing ROSA26-Lox-Stop-Lox-tdTomato (Ai9) mice (28) (Jackson) to a strain expressing Cre recombinase in the early embryo (EIIA-Cre), which was subsequently bred out.

\section{Cell transfer}

Spleens and lymph nodes were forced through a 40 or $70 \mu \mathrm{m}$ mesh into complete RPMI media (Gibco) with $10 \%$ serum. Resting B cells and $\mathrm{CD}^{+} \mathrm{T}$ cells were obtained by magnetic cell sorting (MACS) by using CD43 beads and the CD4 cell negative isolation kit (Miltenyi) respectively, according to the manufacturer's instructions.

\section{Immunizations}

To generate primary GCs, 5-10-week-old C57BL/6 recipient mice were immunized subcutaneously with $10 \mu \mathrm{g} /$ footpad of NP-OVA ( $\mathrm{NP}_{11}$-OVA, $\mathrm{NP}_{14}$-OVA or $\mathrm{NP}_{18}$-OVA, used interchangeably), $\mathrm{NP}_{28}-\mathrm{KLH}$ or $\mathrm{NP}_{23}$-CGG (Biosearch Technologies) precipitated in alum (Imject ${ }^{\circledR}$ Alum, Thermo Scientific) at a 2:1 antigen(PBS):alum ratio in a $25 \mu \mathrm{l}$ volume. For secondary GC generation (photoactivation experiments) and for T cell invasion experiments, mice were primed, i.p., with $50 \mu \mathrm{g}$ of OVA (Grade V, Sigma) precipitated in alum at a 2:1 antigen(PBS):alum ratio in a $100 \mu 1$ volume. 2-6 weeks later, mice were boosted with $25 \mu \mathrm{g}$ of $\mathrm{NP}_{14}$ - or $\mathrm{NP}_{18}$-OVA (Biosearch Technologies) in hind footpads, and popliteal LN were either imaged or harvested for flow cytometry. aDEC-OVA and aDECCS were produced as chimeric antibodies in 293T cells as described (29), and $3 \mu \mathrm{g}$ of recombinant antibody were injected into the footpads of mice 6 days after induction of T cell invasion. 


\section{Intravital imaging}

Intravital imaging was conducted essentially as described previously $(3,4)$. Mice were anaesthetized with $100 \mathrm{mg}$ ketamine, $15 \mathrm{mg}$ xylazine and $2.5 \mathrm{mg}$ acepromazine per kg body weight and were kept anaesthetized by inhalation of $1.25 \%$ isofluorane in $100 \%$ oxygen. Hind legs were shaved using a double-edged razor blade, mice were restrained on a stage warmer at $37^{\circ} \mathrm{C}$ (BioTherm Micro S37; Biogenics) and an incision was made behind the knee of one hind leg. The popliteal LN was exposed and restrained using a metal strap, and the mouse was placed under the microscope objective, fitted with an objective heater set at $40{ }^{\circ} \mathrm{C}$. For follicular dendritic cell labeling, red fluorescent protein tdTomato and yellow fluorescent protein YPet were produced in E. coli and conjugated to NP as described (3), and mice were injected subcutaneously with $1 \mu \mathrm{g}$ and $5 \mu \mathrm{g}$ per footpad of NP-tdTomato and NP-YPet, respectively, one day prior to imaging. For photoactivation experiments, lymph nodes containing PAGFP cells were photoactivated by scanning with a femtosecond-pulsed multiphoton laser tuned to $820-830 \mathrm{~nm}$ wavelength, and then imaged at $940 \mathrm{~nm}$ wavelength, as described in reference 3 . For intravital experiments, cell motility was monitored immediately after photoactivation and at later time points as a measure of viability. For long-term photoactivation experiments, the incision was closed after photoactivation by suturing, and mice were allowed to recover for $17-20 \mathrm{~h}$. After this period, the photoactivated lymph node was explanted and imaged as described previously (15). The half-life of photoactivatable GFP in vivo in non-dividing cells is roughly 30 hours (3). For analysis of $\mathrm{T}_{\mathrm{FH}}$ markers or cell cycle stage by flow cytometry, we photoactivated $\mathrm{PAGFP}^{+}$cells inside GCs (labeled with $\mathrm{CFP}^{+} \mathrm{B} 1-8^{\text {hi }}$ cells or NP-tdTomato, respectively), in the T cell zone (labeled by injection of $\mathrm{DsRed}^{+}$or tdTomato ${ }^{+}$naïve T cells), or in B cell follicles (between GCs and T cell zone) in explanted LN. All imaging experiments were carried out using Olympus BX61 upright microscopes (Olympus 25× 1.05 NA Plan objective), fitted with either a Coherent Chameleon Vision II laser (Rockefeller University Bio-Imaging Resource Center) or a SpectraPhysics MaiTai DeepSee laser (Victora lab, Whitehead Institute).

\section{Image acquisition and analysis}

Images were acquired using 920-940 $\mathrm{nm}$ excitation and the following filters: a first pair of overlapping CFP (460-510) and GFP (495-540) filters, separated by a 505 dichroic mirror, which allowed maximal signal detection for GFP, PAGFP, CFP, and YPet; $2^{\text {nd }}$ harmonics (collagen) were also detected in the CFP filter at 930-40 nm excitation; and a third filter (575-630) for the red signal (tdTomato, DsRed). For whole-mounted LN reconstructions, we acquired tiles of $250 \mu \mathrm{m}$-deep Z-stacks (corresponding to the cortical half of the $\mathrm{LN}$ ) with 5 or $10 \mu \mathrm{m} \mathrm{Z}$ resolution, and an X-Y resolution of $320 \times 320$ or $512 \times 512$ pixels per tile (1.325 $\mu \mathrm{m}$ per pixel) (15). GFP OT-II cell density in whole-LN scans was quantified using Imaris 7.3.1 software (Bitplane AG). GC and LN boundaries were defined with the Surfaces feature, and $\mathrm{GFP}^{+}$cells were counted using the Spots automatic detection feature. $\mathrm{T}$ cell density ratio between GCs and the remainder of the $\mathrm{LN}$ was calculated as (GC T cells/ GC volume)/[(total T cells - GC T cells)/(LN volume - GC volume $)]$. The positions of photoactivated cells and of CFP/GFP/DsRed T cells in multiple color experiments were determined manually, slice by slice, by combining the Spots and Ortho-slicer tools in Imaris. Photoacitvated cells detected outside of the originally photoactivated area (defined as the GC with the highest density of photoactivated cells) either in the follicles or neighboring GCs were counted as emigrating $\mathrm{T}$ helper cells. Movies were acquired as $40 \mu \mathrm{m} Z$-stacks with $5 \mu \mathrm{m} \mathrm{Z}$ resolution and $512 \times 512 \mathrm{X}$-Y resolution ( 0.4 to $0.8 \mu \mathrm{m} /$ pixel, depending on magnification). Time resolution was $\sim 30$ s per frame. Movies are presented at approximately 300x real time. 


\section{Flow cytometry}

Lymph nodes were forced through a 40 or $70 \mu \mathrm{m}$ mesh into complete RPMI with $10 \%$ fetal calf serum. Single-cell suspensions thus obtained were pretreated for $5^{\prime}$ with $1 \mu \mathrm{g} / \mathrm{ml}$ of antiCD16/32 (2.4G2, Bio-X-Cell) and then stained for $30 \mathrm{~min}$ at $4{ }^{\circ} \mathrm{C}$ in PBS supplemented with $2 \%$ FCS and $1 \mathrm{mM}$ EDTA using the antibodies indicated in Table S1. For cell cycle analysis, cells were fixed and permeabilized using the Cytofix/CytopermTM kit (BD) and stained with $10 \mu \mathrm{g} / \mathrm{ml}$ DAPI (Invitrogen) for 3 minutes. Samples were analyzed on BD Fortessa or BD LSR-I flow cytometers (BD). T cells were gated as $\mathrm{CD}^{+}$and CD45. $1^{+}$, for PAGFP OT-II analysis, or $\mathrm{CD} 4^{+}, \mathrm{TCR}^{+}, \mathrm{CD} 44^{\text {high }}, \mathrm{CD} 62 \mathrm{~L}^{\text {low }}$ for polyclonal PAGFP T cell analysis. GC B cells were gated as live/single, B220 $0^{+}$, FAS ${ }^{\text {high }}$, CD38 $8^{-}$. Plasmablasts/ plasma cells were gated as live/single, B220 ${ }^{\text {int }}, \mathrm{CD} 138^{+}$.

To test whether germinal center $\mathrm{T}$ cells egress entirely from lymph nodes, we adoptively transferred OT-II PAGFP ${ }^{+}$CD $45.1^{+}$CD4 T cells and B1- $8^{\text {hi }}$ CD $45.1^{+} \mathrm{B}$ cells into OVAprimed wild type (CD45.2 $2^{+}$) mice and boosted the hind footpads subcutaneously with NPOVA. At day 6 of the GC response, 3-6 GCs in the draining lymph nodes were photoactivated in living mice. After 36 hours, mice were sacrificed and cells from blood, spleen and LN (excluding the photoactivated LN), were labeled with anti-CD45.1-PE followed by anti-PE magnetic beads. CD 45.1 $1^{+}$cells from each individual mouse were then enriched by binding to magnetized (MACS) columns. Eluted cells were analyzed by flow cytometry for detection of photoactivated $\mathrm{CD} 45.1^{+} \mathrm{T}$ cells. $\mathrm{T}$ cells were pre-gated as $\mathrm{B} 220^{-}$, MHC-II ${ }^{-}, \mathrm{CD} 11 \mathrm{C}^{-}, \mathrm{CD} 11 \mathrm{~b}^{-}, \mathrm{F} 4 / 80^{-}, \mathrm{CD}^{+}, \mathrm{CD}^{+}$.

\section{Lymph node microdissection}

Popliteal lymph nodes were photoactivated in vivo as described above (Intravital imaging), with the exception that $3 \times 10^{6} \mathrm{PAGFP}^{+} \mathrm{B} 1-8^{\text {hi }} \mathrm{B}$ cells were transferred 24 hours prior to boost to increase signal intensity in the photoactivated GC. Lymph nodes were explanted either immediately or 24 hours after photoactivation. Explanted lymph nodes were mounted between 2 coverslips and scanned by TPLSM to identify the original photoactivated GC (defined as the GC with the highest density of photoactivated cells). The original GC was photoactivated once more at high laser power to increase visibility, and the LN was cut into two fragments using a \#10 disposable scalpel (Miltex) under a Leica MZFLIII fluorescence stereomicroscope fitted with a PlanAPO 1.0 X objective. Both parts of the lymph node were processed and stained in separate, as described above (Flow cytometry).

\section{Supplementary Material}

Refer to Web version on PubMed Central for supplementary material.

\section{Acknowledgments}

We thank H. Ploegh, K. Strijbis and H. Sive for mice and equipment use, and E. Browne for helpful discussion. The data presented in the paper are tabulated in the main paper and in the supplementary materials. ZS is a Human Frontiers of Science fellow. GP is a Swiss National Science Foundation fellow. ADG was supported by NIH Medical Scientist Training Program grant T32GM07739 to the Weill Cornell/Rockefeller/Sloan-Kettering TriInstitutional MD-PhD Program. Support for the Rockefeller University multiphoton microscope was granted by the Empire State Stem Cell Fund through NYSDOH Contract \#C023046. This work was supported in part by NIH grant 5DP5OD012146-02 and MOD Basil O'Connor Award to GDV and by NIH grants AI037526-19, AI072529-06 and AI100663-01 to MCN. MCN is an HHMI investigator.

\section{References and Notes}

1. Vinuesa CG, et al. A RING-type ubiquitin ligase family member required to repress follicular helper T cells and autoimmunity. Nature. May 26.2005 435:452. [PubMed: 15917799] 
2. Allen CD, Okada T, Tang HL, Cyster JG. Imaging of germinal center selection events during affinity maturation. Science. Jan 26.2007 315:528. [PubMed: 17185562]

3. Victora GD, et al. Germinal Center Dynamics Revealed by Multiphoton Microscopy with a Photoactivatable Fluorescent Reporter. Cell. Nov 12.2010 143:592. [PubMed: 21074050]

4. Schwickert TA, et al. A dynamic T cell-limited checkpoint regulates affinity-dependent B cell entry into the germinal center. J Exp Med. May 16.2011

5. Dominguez-Sola D, et al. The proto-oncogene MYC is required for selection in the germinal center and cyclic reentry. Nature immunology. Nov.2012 13:1083. [PubMed: 23001145]

6. Victora GD, Nussenzweig MC. Germinal centers. Annual review of immunology. 2012; 30:429.

7. Vinuesa CG, Cyster JG. How T cells earn the follicular rite of passage. Immunity. Nov 23.2011 35:671. [PubMed: 22118524]

8. Crotty S. Follicular helper CD4 T cells (TFH). Annu Rev Immunol. Apr 23.2011 29:621. [PubMed: 21314428]

9. King C, Tangye SG, Mackay CR. T Follicular Helper (T(FH)) Cells in Normal and Dysregulated Immune Responses. Annu Rev Immunol. 2008; 26:741. [PubMed: 18173374]

10. Qi H, Cannons JL, Klauschen F, Schwartzberg PL, Germain RN. SAP-controlled T-B cell interactions underlie germinal centre formation. Nature. Oct 9.2008 455:764. [PubMed: 18843362]

11. Fazilleau N, Mark L, McHeyzer-Williams LJ, McHeyzer-Williams MG. Follicular helper T cells: lineage and location. Immunity. Mar 20.2009 30:324. [PubMed: 19303387]

12. Deenick EK, et al. Follicular helper T cell differentiation requires continuous antigen presentation that is independent of unique B cell signaling. Immunity. Aug 27.2010 33:241. [PubMed: 20691615]

13. Choi YS, et al. ICOS receptor instructs $\mathrm{T}$ follicular helper cell versus effector cell differentiation via induction of the transcriptional repressor Bcl6. Immunity. Jun 24.2011 34:932. [PubMed: 21636296]

14. Supplementary materials and methods available at Science Online.

15. Liu K, et al. In vivo analysis of dendritic cell development and homeostasis. Science. Apr 17.2009 324:392. [PubMed: 19286519]

16. Garside P, et al. Visualization of specific B and T lymphocyte interactions in the lymph node. Science. Jul 3.1998 281:96. [PubMed: 9651253]

17. Kroese FG, Wubbena AS, Seijen HG, Nieuwenhuis P. Germinal centers develop oligoclonally. Eur J Immunol. Jul.1987 17:1069. [PubMed: 3301368]

18. Liu YJ, Zhang J, Lane PJ, Chan EY, MacLennan IC. Sites of specific B cell activation in primary and secondary responses to T cell-dependent and T cell-independent antigens. Eur J Immunol. Dec.1991 21:2951. [PubMed: 1748148]

19. Yu D, Vinuesa CG. The elusive identity of T follicular helper cells. Trends Immunol. Oct.2010 31:377. [PubMed: 20810318]

20. Germain RN, Miller MJ, Dustin ML, Nussenzweig MC. Dynamic imaging of the immune system: progress, pitfalls and promise. Nat Rev Immunol. Jul.2006 6:497. [PubMed: 16799470]

21. Kuppers R, Zhao M, Hansmann ML, Rajewsky K. Tracing B cell development in human germinal centres by molecular analysis of single cells picked from histological sections. Embo J. Dec 15.1993 12:4955. [PubMed: 8262038]

22. Zheng B, Han S, Kelsoe G. T helper cells in murine germinal centers are antigen-specific emigrants that downregulate Thy-1. The Journal of experimental medicine. Sep 1.1996 184:1083. [PubMed: 9064325]

23. Roers A, Hansmann ML, Rajewsky K, Kuppers R. Single-cell PCR analysis of T helper cells in human lymph node germinal centers. The American journal of pathology. Mar.2000 156:1067. [PubMed: 10702422]

24. Golby SJ, Dunn-Walters DK, Spencer J. Human tonsillar germinal center T cells are a diverse and widely disseminated population. European journal of immunology. Nov.1999 29:3729. [PubMed: 10556829]

25. Shih TA, Roederer M, Nussenzweig MC. Role of antigen receptor affinity in T cell-independent antibody responses in vivo. Nat Immunol. Apr.2002 3:399. [PubMed: 11896394] 
26. Guo M, et al. A monoclonal antibody to the DEC-205 endocytosis receptor on human dendritic cells. Hum Immunol. Aug.2000 61:729. [PubMed: 10980384]

27. Boes M, et al. T-cell engagement of dendritic cells rapidly rearranges MHC class II transport. Nature. Aug 29.2002 418:983. [PubMed: 12198548]

28. Madisen L, et al. A robust and high-throughput Cre reporting and characterization system for the whole mouse brain. Nature neuroscience. Jan.2010 13:133.

29. Boscardin SB, et al. Antigen targeting to dendritic cells elicits long-lived T cell help for antibody responses. J Exp Med. Mar 20.2006 203:599. [PubMed: 16505139] 

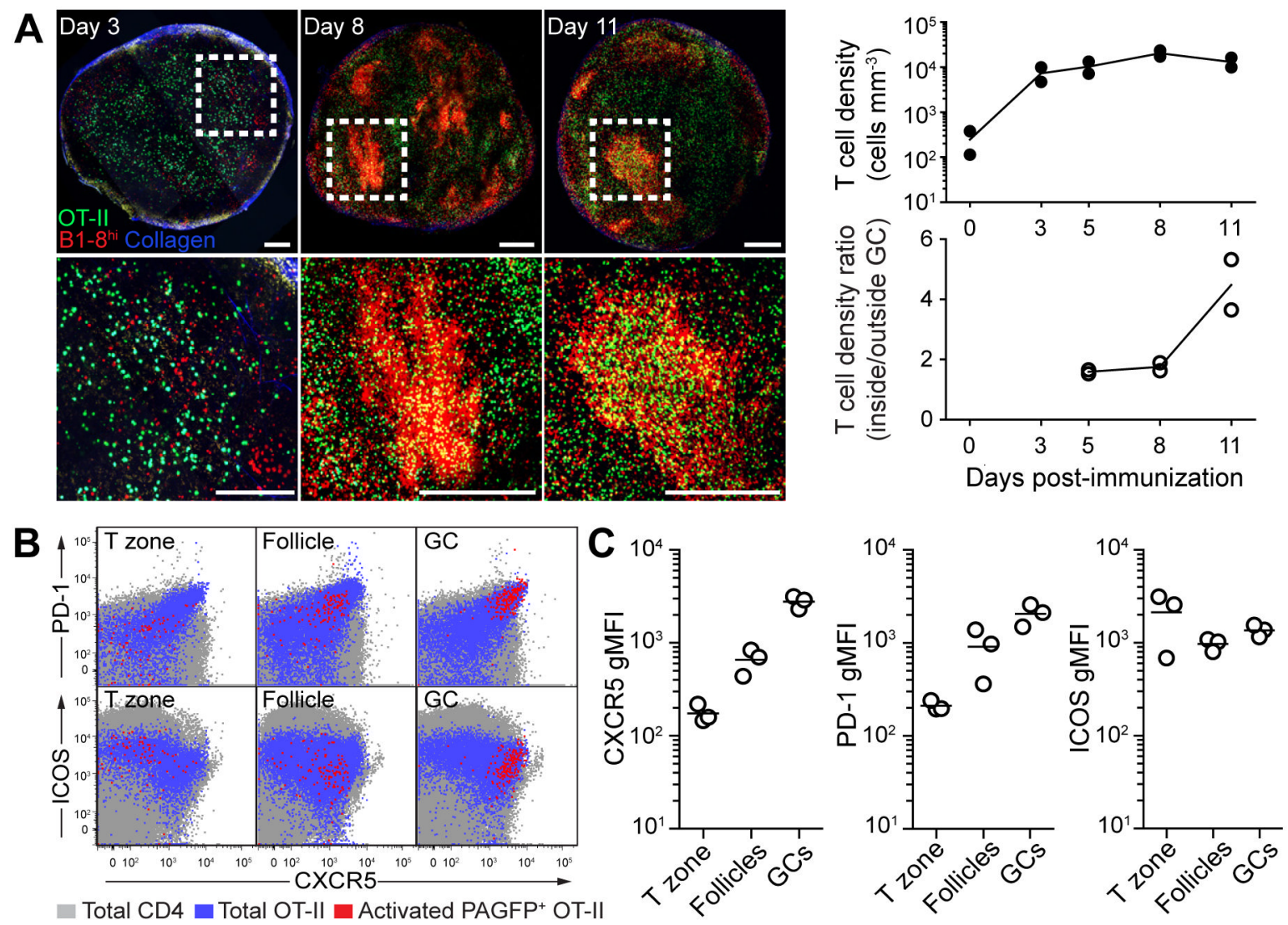

Figure 1. Expansion kinetics and anatomical distribution of $\mathbf{T}_{\mathbf{F H}}$ during germinal center formation

(A) $3 \times 10^{4} \mathrm{GFP}^{+}$OVA-specific T cells (OT-II) and $1.5 \times 10^{6}$ tdTomato $^{+} \mathrm{B} 1-8^{\text {hi }} \mathrm{B}$ cells (corresponding to $1.5 \times 10^{5} \mathrm{Ig} \lambda^{+}, \mathrm{NP}$-specific B cells) were transferred into wild type mice 1 $\mathrm{d}$ before subcutaneous immunization with NP-OVA in alum. Popliteal LN were explanted at $0,3,5,8$, and 11 days after immunization and subjected to whole-lymph node scanning by TPLSM. Collapsed Z-stacks (50-100 $\mu \mathrm{m}$ total volume, $5 \mu \mathrm{m}$ steps) are shown. Scale bars, $300 \mu \mathrm{m}$. Right: quantitation of $\mathrm{T}$ cell density and density ratio (T cells in GC/T cells outside of GC) in two independent experiments. (B-C) Expression of $\mathrm{T}_{\mathrm{FH}}$ markers on PAGFP ${ }^{+} \mathrm{OT}$ II cells photoactivated in different LN regions as described in Fig. S1. Each symbol represents one experiment. 
A Day 10 post immunization
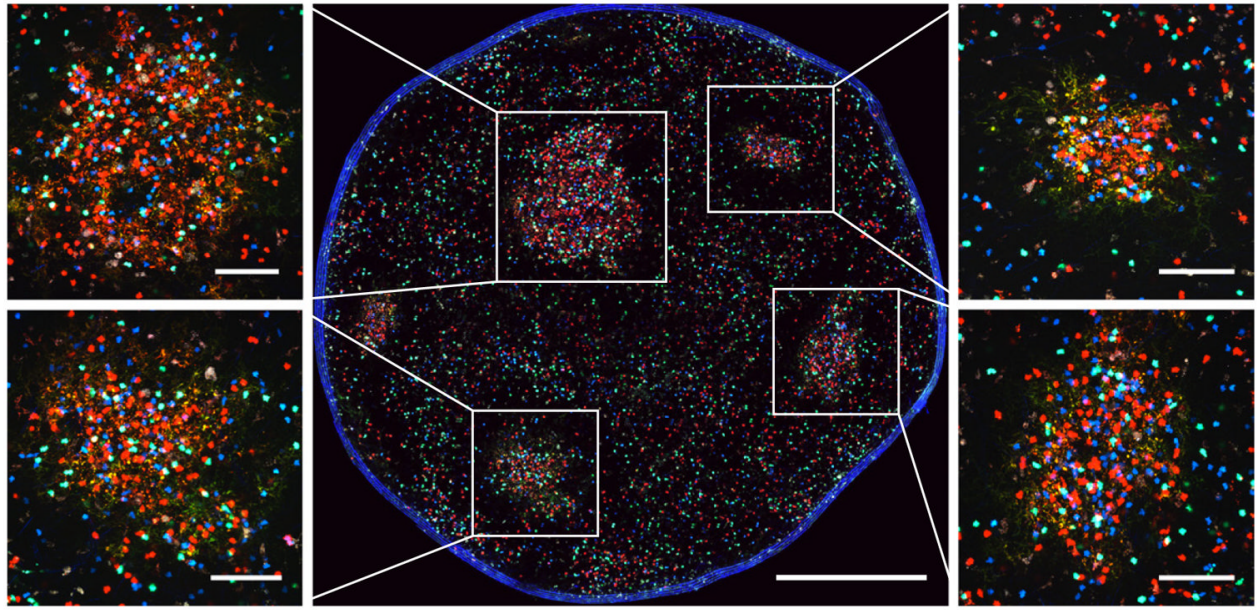

- OT-II-GFP • OT-II-CFP • OT-II-DsRed $\approx$ FDC network (NP-tdTomato + NP-YPet) $\approx$ Collagen $\left(2^{\text {nd }}\right.$ harmonics $)$
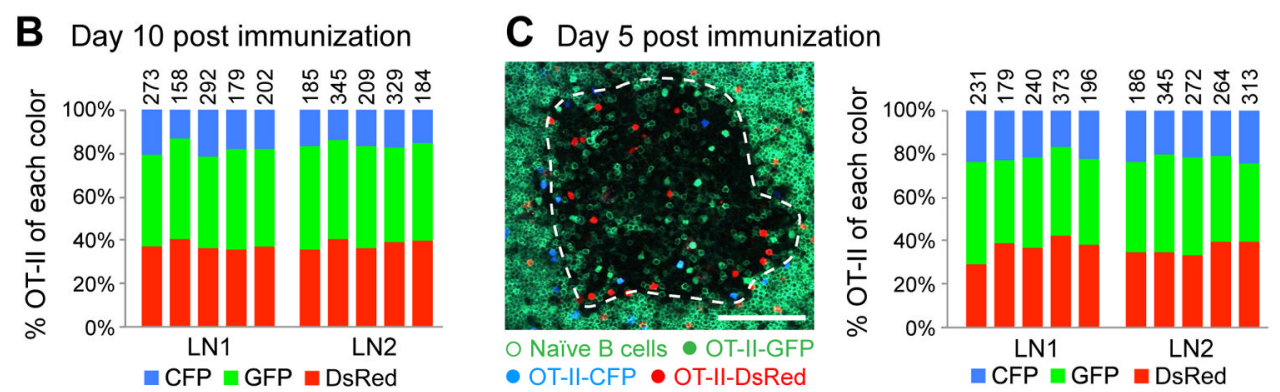

Figure 2. GC- $\mathbf{T}_{\mathbf{F H}}$ cells in individual germinal centers are not clonally restricted

(A) A total of $3 \times 10^{5}$ OT-II cells expressing CFP, GFP or DsRed $\left(\sim 10^{5}\right.$ of each color $)$ and $3-5 \times 10^{6}$ non-fluorescent B1-8 ${ }^{\text {hi }}$ B cells (corresponding to $3-5 \times 10^{5} \operatorname{Ig} \lambda^{+}$, NP-specific cells) were transferred into WT recipients 1 day before subcutaneous immunization with NP-OVA in alum. Draining LN were explanted 10 days later and imaged by TPLSM. FDC networks were labeled by injection of a mixture of NP-tdTomato and NP-YPet (orange). Central image shows a $50 \mu \mathrm{m}$-deep collapsed Z-stack (10 $\mu \mathrm{m}$ steps) of a whole LN containing multiple GCs, magnified in the outer panels. Scale bars, $100 \mu \mathrm{m}$ (large panel); 50 $\mu \mathrm{m}$ (side panels). (B) Quantitation of data as in (A) across multiple LN from different mice. Each bar represents a single GC. Numbers on top of bars indicate the number of T cells counted in each GC. (C) T and B cells as described in (A) were transferred to H-2A $\beta$-GFP mice to allow visualization of early GCs, and LN were imaged 5 days after immunization with NP-OVA in alum. Image of one GC and quantitation of multiple GCs are shown. Scale bar, $100 \mu \mathrm{m}$. Each set of experiments was performed twice (additional images and quantitation in Fig. S3). 
A 20 hours after photoactivation
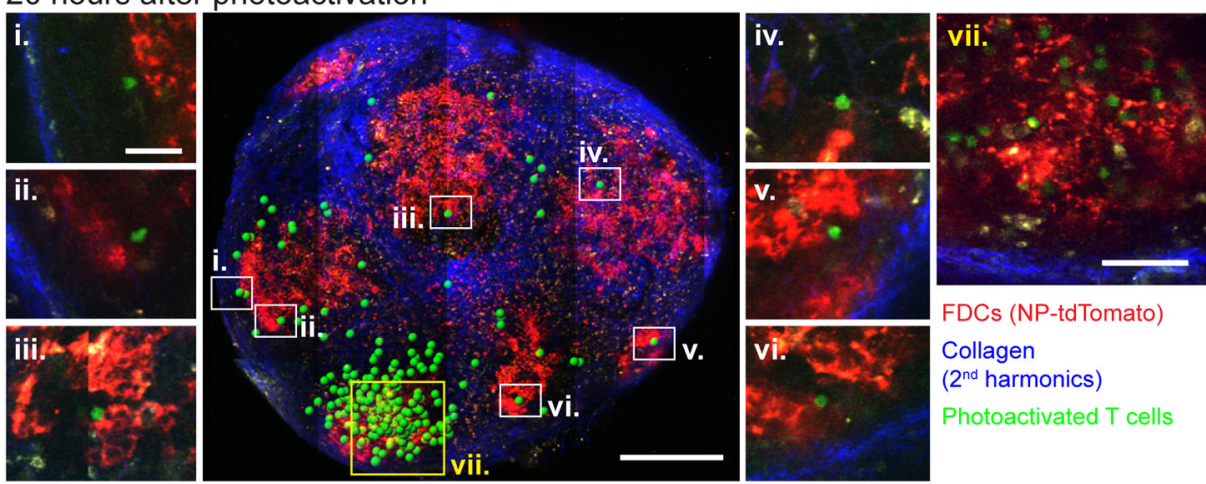

FDCs (NP-tdTomato)

Collagen

( $2^{\text {nd }}$ harmonics)

Photoactivated T cells

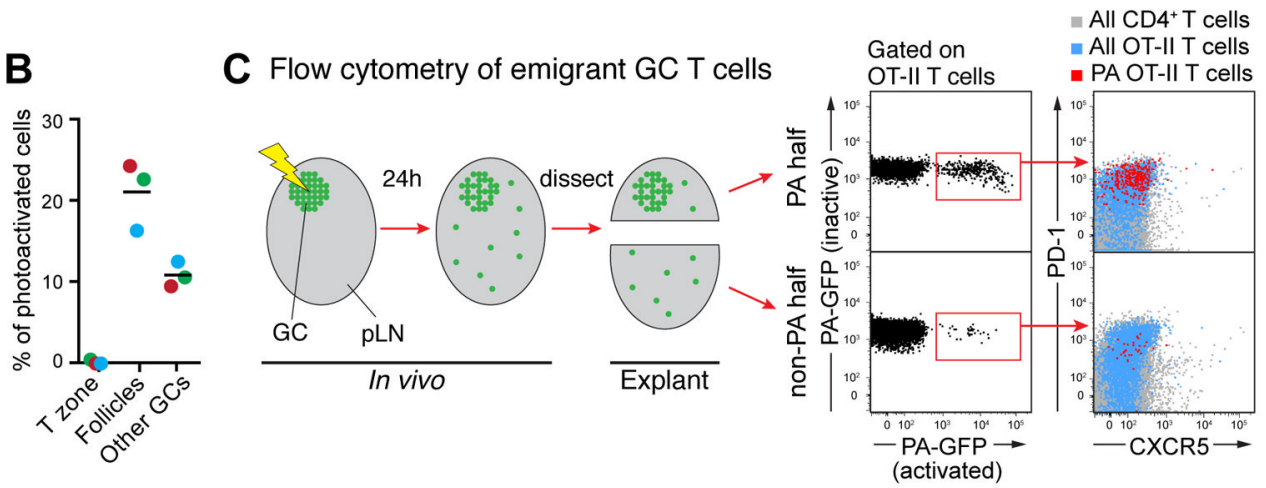

Figure 3. GC-T $\mathbf{F H}_{\mathrm{FH}}$ exchange between neighboring germinal centers

(A) GCs containing OT-II PAGFP ${ }^{+}$T cells were generated as detailed in Fig. S6A, and the LZ of a single GC was photoactivated within a living mouse. Center panel: collapsed Zstack image of the cortical half ( $250 \mu \mathrm{m}$ depth) of a whole-LN scan taken $\sim 20$ hours after photoactivation. Outer panels ( $i-v i)$ : high-magnification images of the regions indicated in the center panel. Panel vii: original photoactivated GC. Scale bars, $300 \mu \mathrm{m}$ (center panel), $20 \mu \mathrm{m}$ (panels i-vi), $30 \mu \mathrm{m}$ (panel vii). Green spots are placed over photoactivated OT-II cells in low-magnification panels to improve visualization. (B) Quantitation of 3 experiments as in (A) (95, 184 and 208 photoactivated cells analyzed in 3 independent experiments). Each color represents one experiment. Average distance covered by photoactivated cells was $334 \mu \mathrm{m}$; the furthest $10 \%$ of cells traveled on average $790 \mu \mathrm{m}$. (C) Single GCs in popliteal lymph nodes containing PAGFP ${ }^{+}$OT-II T cells were photoactivated in vivo as in (A), then explanted and dissected as shown in Fig. S6. FACS plots show PD-1 and CXCR5 expression in emigrant $\mathrm{GC}-\mathrm{T}_{\mathrm{FH}}$ cells (pooled data from 2 mice). Data representative of 5 mice from 3 independent experiments. 


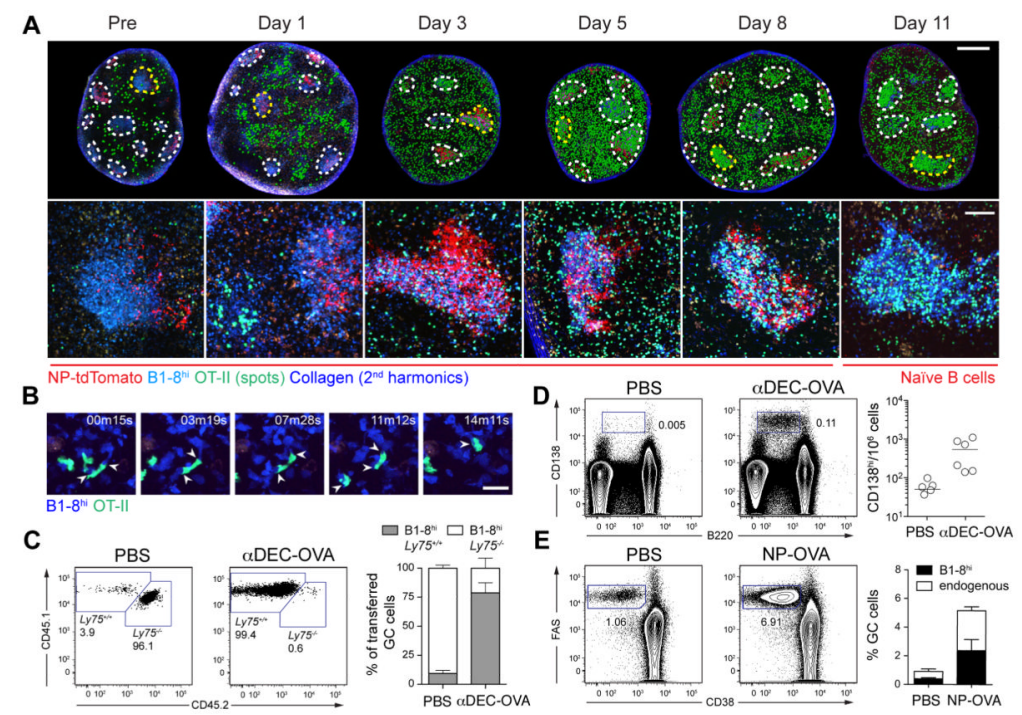

Figure 4. Newly-activated $\mathbf{T}_{\mathbf{F H}}$ can enter ongoing germinal center reactions

(A) Kinetics of invasion of ongoing GCs by newly activated T cells as described in figure S8A. Top: montage showing whole-LN TPLSM scans. Collapsed Z-stacks are $90 \mu \mathrm{m}$ deep (pre and day 1) or $40 \mu \mathrm{m}$ deep (days 3-11). GCs containing $\mathrm{CFP}^{+} \mathrm{B} 1-\mathrm{g}^{\mathrm{hi}} \mathrm{B}$ cells (blue) and NP-tdTomato-coated FDCs (red) are circled with dotted lines. Green spots are placed over $\mathrm{GFP}^{+} \mathrm{T}$ cells to improve visualization. Background outside LNs was deleted. Bottom: higher-magnification images of GCs circled in yellow in the top panel. Scale bars, $0.5 \mathrm{~mm}$ (top), $100 \mu \mathrm{m}$ (bottom). (B) Time series showing cognate T-B interactions in an invaded GC on day 11 post-boost. Scale bar, $20 \mu \mathrm{m}$. (C-D) Recipient mice were treated with aDEC205OVA or PBS 6 days after NP-OVA boost as outlined in figure S8D. Flow cytometry plots showing proportion of $L y 75^{+/+}$and $L y 75^{-/-}$B $1-8^{\text {hi }}$ GC B cells $(\mathbf{C})$ or percentage of plasmablasts among all single cells (D) 3 days after aDEC205-OVA treatment. (E) Flow cytometry plots comparing the proportion of $\mathrm{GC}$ cells $\left(\mathrm{Fas}^{+} \mathrm{CD} 38^{-}\right)$in mice treated as outlined in figure S8E or receiving PBS instead of the NP-OVA boost at day 0. Mean \pm SEM of 2-3 experiments is depicted in the right-most panels. 